\title{
An Analysis of Asymptotic Properties and Error Control under the Exponential Jump-Diffusion Model for American Option Pricing
}

\author{
Mohamed Maidoumi $\mathbb{D}$, Boubker Daafi, and Mehdi Zahid \\ LAMAI, Cadi Ayyad University, Marrakech, Morocco \\ Correspondence should be addressed to Mohamed Maidoumi; maidoumi-22@hotmail.fr
}

Received 23 June 2021; Accepted 21 August 2021; Published 28 September 2021

Academic Editor: Igor Andrianov

Copyright (C) 2021 Mohamed Maidoumi et al. This is an open access article distributed under the Creative Commons Attribution License, which permits unrestricted use, distribution, and reproduction in any medium, provided the original work is properly cited.

\begin{abstract}
Our work is aimed at modeling the American option price by combining the dynamic programming and the optimal stopping time under two asset price models. In doing so, we attempt to control the theoretical error and illustrate the asymptotic characteristics of each model; thus, using a numerical illustration of the convergence of the option price to an equilibrium price, we can notice its behavior when the number of paths tends to be a large number; therefore, we construct a simple estimator on each slice of the number of paths according to an upper and lower bound to control our error. Finally, to highlight our approach, we test it on different asset pricing models, in particular, the exponential Lévy model compared to the simple Black and Scholes model, and we will show how the latter outperforms the former in the real market (Microsoft "MSFT" put option as an example).
\end{abstract}

\section{Introduction}

The first academic work on pricing options, in particular that of Black and Scholes [1], has allowed the development of derivatives markets. But the use of this theory or those which followed for the evaluation of negotiated financial contracts and related hedging strategies deduced could not be done in practice without the choice of a model and its estimate. Today, it is admitted, on the basis of numerous empirical studies, that the Gaussian hypothesis for the log return on financial assets has reduced validity (see Section 2.3.1). The reader will find examples in the article [2] as well as in [3]. The first alternative models to the Gaussian model were the stable model proposed by $[4,5]$, and within the framework of option valuation, Merton [6] is the first author to have developed a non-Gaussian model; let us note that for a long time, evaluating American option has been considered inadequate with the traditional forward Monte Carlo simulations; hence, the binomial tree model [7] and finite difference [8] methods were used as the only numerical method before the mid-1990s when new methods appeared.
The most popular method was proposed by Carriere [9] using optimal stopping times and then developed, improved, and popularized by Longstaff and Schwartz [10] by combining between least square regression and Monte Carlo simulation; for a more comprehensive review of the literature, the reader may profitably consult the works of Tankov et al. [11]. In this paper, we compare between the models of Black and Scholes in 1973 [1] and an exponential diffusion-jump model, but when using a modeling process using discontinuous trajectories, a delicate problem arises, which is the incompleteness of markets, and the riskneutral measure is not unique. Numerous works such as those of Fujiwara and Miyahara [12] or Tankov et al. [11] have been devoted to this subject. Here, we adopt a more direct point of considering view that the dynamic followed by the price of financial assets is given in a risk-neutral universe.

The main advantage of Monte Carlo simulation resides in its convergence rate which does not depend on the number of the underlying, and it is useful thanks to its ease of handling a large range of models. However, Monte Carlo 
simulations stay slow and their standard form is not suitable for American options; the reason comes from the fact that their trajectories dissociate (date of execution of the option is independent of each trajectory); in fact, the possibility of exercising at any time requires calculating on each trajectory the expected value of the option which is a conditional expectation. [10] proposes an algorithm that requires rejection of certain trajectories (trajectories that are out of the money) and compares the intrinsic value (discounted payoff) to be able to decide whether to execute the option or keep it. This practical term is not feasible even if we limit ourselves to a finite number of possible exercise dates in order to solve a dynamic programming of the optimal exercise policy that requires backward simulations.

In Section 1, we present the theoretical framework of the Longstaff and Schwartz algorithm and we show the effectiveness of this approach compared to the dynamic algorithm of Tsitsiklis and van Roy. In Section 2, we focus on the numerical implementation of the Longstaff and Schwartz algorithm and compare the B\&S model and Lévy jump-diffusion. We also focus on the theoretical and asymptotic convergence of this algorithm, and towards the end, we present our method for controlling the error using a put option on Microsoft "MSFT" data as an example from "Yahoo Finance" and we implement the validation of these models in a real market.

\section{Pricing American Option Theoretical Framework}

We consider an American option of value $U(t, Y(t))$ which depends on the time and $m$ factors $Y(t)=Y_{1}(t), \cdots, Y_{m}(t)$; for example, an option in the B\&S model depends on one factor $Y_{1}(t)=S(t)$; in the case of exponential Lévy jumpdiffusion model, the option depends, respectively, on the underlying price, jump size, and number of jumps in $[0, T] Y$ $(t)=\left(Y_{1}(t)=S(t), Y_{2}(t)=J_{t}, Y_{3}(t)=N_{t}\right)$. We are looking for the empirical distribution of the probability of $U(t, Y(t))$ or its moments, and we are interested in all values of $U(t, Y$ $(t))$ in the meantime $[0, T]$ (American option).

Monte Carlo simulation is a probabilistic method used in the case where the distribution law $U(t, Y(t))$ or the first moments are not defined analytically. This simulation consists in generating a large number of possible trajectories $\left.S_{t}\right|_{t=t_{1} \cdots t=t_{N}}$; therefore, we build an empirical distribution of $S(t)$.

2.1. Characterization of the Optimal Stopping Time Policy by the Snell Envelope. In this section, we aim to explain how the Snell envelope provides an introduction of optimal stopping time theory for pricing an American option.

Definition 1. Let $\left(\Omega, F,\left(\mathscr{F}_{t}\right)_{)_{t \in\{0, \cdots, T\}}}, P\right)$ be a filtered probability space, which means we discretize the time space $[0, T]$ into $n$ pieces and we consider $\Omega$ the measure space of the universe of possible values and $F \subset \Omega$ is a $\sigma$-algebra of the set's events; $P$ is the probability measure on $F$; $\mathscr{F}_{t}$ is the natural filtration on $(\Omega, F)$; i.e., $\forall t_{i}, t_{j} \in\{0, \cdots, T\}, \mathscr{F}_{t_{i}}$ is a $\sigma$ -algebra and if $i<j, \mathscr{F}_{t_{i}} \subset \mathscr{F}_{t_{j}} ; T$ is the time horizon, and the underlying $\left(S_{t}\right)_{t \in\{0, \cdots, T\}}$ is defined on the filtered probability space with a finite time horizon $T$.

Let $\left(O_{t_{k}}\right)_{k=0 \cdots n}$ be an adapted and integrable random variable. The Snell envelope of the process $\left(O_{t_{k}}\right)_{k=1 \cdots n}$ is defined as

$$
U_{t_{j}}=\operatorname{ess} \sup _{\tau \in \Gamma_{t_{j}}} \mathbf{E}\left(O_{\tau} \mid \mathscr{F}_{t_{j}}\right), \quad j=1 \cdots n,
$$

where $\Gamma_{t_{j}}$ is the set of all stopping time in $\left\{t_{j}, \cdots, T\right\}$.

Proposition 2. The Snell envelope $\left(U_{t_{k}}\right)_{t_{k}=0 \cdots, t_{n}=T}$ of $\left(O_{t_{k}}\right)_{t_{k}=0 \cdots t_{n}=T}$ fulfills these properties:

(1) $U_{T}=O_{T}$

(2) $U_{t_{k}}=\max \left\{O_{t_{k}}, E\left(U_{t_{k+1}} \mid F_{t_{k}}\right)\right\}$ such that $k=0, \cdots, n$

(3) $\left(U_{t_{k}}\right)_{k=1 \cdots n}$ is the smallest supermartingale dominat$\operatorname{ing}\left(O_{t_{k}}\right)_{k=1 \cdots n}$

Proof. See Appendix A. $\square \square$

The optimal time is defined as follows:

Definition 3. $\tau$ is an optimal time iff $\mathbf{E}\left[O_{\tau} \mid \mathscr{F}_{0}\right]=\sup _{\tau}{ }^{\prime} \in \Gamma_{0} \mathbf{E}\left[O_{\tau} \mid \mathscr{F}_{0}\right]$.

Using the Snell envelope, we can characterize the optimal stopping time policy to evaluate an American option.

Theorem 4. A stopping time $\tau$ is optimal if and only if

(i) $U_{\tau}=O_{\tau}$

(ii) $U_{\tau \wedge t_{j}}$ is a martingale

Proof. See Appendix B. $\square \square$

Proposition 5. Let $\tau=\min \left\{k \geq 0 \mid U_{k}=O_{k}\right\}$; hence, $\tau$ is an optimal stopping time.

Proof. See Appendix C. $\square \square$

2.2. Effectiveness of Longstaff and Schwartz Policy Iteration Approach. We consider the same filtered probability space $\left(\Omega, F,\left(\mathscr{F}_{t}\right)_{0 \leq t \leq T}, \mathbb{P}\right), T \geq 0$, and $W_{t}=\left(W_{t}^{1}, \cdots, W_{t}^{d}\right)$, a Brownian motion of $d$ dimension. We notice $\left(O_{t_{k}}\right)_{k=1 \cdots N}$ as the payoff process and $\left(U_{t_{k}}\right)_{k=1 \cdots N}$ as the discounted value of the option (Snell envelope) $U_{t_{k}}=\operatorname{ess} \sup _{\tau \in \Gamma_{t_{k}}} E\left[O_{\tau} \mid \mathscr{F}_{t_{k}}\right]$ such that $\Gamma_{t_{k}}$ is the set of all $\mathscr{F}_{t}$-stopping time in $t \in\left\{t_{k}, \cdots, t_{N}\right.$ $=T\}$; we aim to approximate $U_{0}$.

The Longstaff and Schwartz algorithm uses the same trajectories for each step due to its dynamic programming that 
depends on the stopping time instead of the value function as in the case of Tsitsiklis and van Roy [13]. This approach is very advantageous because it is robust, has low computational burden, and saves time.

$$
\left\{\begin{array}{l}
\tau_{N}=t_{N}, \\
\tau_{k}=t_{k} \mathbf{1}_{O_{t_{k}} \geq E\left[O_{t_{k+1}} \mid \mathscr{F}_{t_{k}}\right]}+\tau_{k+1} \mathbf{1}_{O_{t_{k}} \leq E\left[O_{t_{k+1}} \mid \mathscr{F}_{t_{k}}\right]},
\end{array}\right.
$$

such that $\tau_{k}$ is the smallest optimal stopping time after $t_{k}$; the first line of the system (2) highlights the fact that the Snell envelope is equal to the payoff at time $T$. The second line is a comparison between the payoff at time $t_{k}$ and the conditional expectation of the payoff at time $t_{k+1}$ on $\mathscr{F}_{t_{k}}$ that we design by continuation value. Now, the main challenge that arises is to approximate conditional expectation.

\subsection{Longstaff and Schwartz Approach and Monte Carlo} Simulation

Theorem 6. Assume that $\left(X_{t}\right)_{t=0 \ldots T}$ is a Markovian process and $\phi_{k}$ is a $\mathscr{F}_{t}$ measurable function; then,

$$
E\left[O_{t_{k+1}} \mid \mathscr{F}_{t_{k}}\right]=E\left[O_{t_{k+1}} \mid X_{t_{k}}\right]=\phi_{k}\left(X_{t_{k}}\right)
$$

Remark 7. Because of the orthogonality of conditional expectation in $L^{2}$, we can compute $\phi_{k}$ by the least square:

$$
\phi_{k}=\inf _{\phi \in \Phi} E\left[\left|O_{t_{k+1}}-\phi\left(X_{t_{k}}\right)\right|^{2}\right]
$$

where $\Phi$ is the set of $L^{2}\left(\sigma\left(X_{t_{k}}\right)\right)$ functions.

The classical numerical (LS) algorithm is based on the approximation of conditional expectation by a finite $p$ -dimensional vector (polynomial basis, logistic.) in the $L^{2}$ space. We notice $\left(\varphi_{k}\right)_{k=1, \cdots, p}$ this finite vector such that it is an $L^{2}$ basis and $\phi_{k}^{p}\left(X_{t_{k}}, \theta_{k}\right)=\sum_{n=1}^{p} \theta_{n} \varphi_{n}\left(X_{t_{k}}\right)$.

(i) Let $\left\{S_{t_{0}}^{(m)}, \cdots, S_{t_{N}}^{(m)}\right\}$ be the price paths and $\left\{O_{t_{0}}^{(m)}, \cdots\right.$, $\left.O_{t_{N}}^{(m)}\right\}$ the payoff paths, such that $m \in\{1, \cdots, M\}$. We present the LS algorithm with Monte Carlo approximation policy as

$$
\left\{\begin{array}{l}
\widehat{\tau}_{N}^{p,(m)}=t_{N}, \\
\widehat{\tau}_{k}^{p,(m)}=t_{k} \mathbf{1}_{O_{t_{k}}^{(m)} \geq \phi_{k}^{p}\left(s_{t_{k}}^{(m)}, \hat{\theta}_{k}^{p, M}\right)}+\tau_{k+1} \mathbf{1}_{O_{t_{k}}^{(m)} \leq \phi_{k}^{p}\left(S_{t_{k}}^{(m)}, \widehat{\theta}_{k}^{p, M}\right)},
\end{array}\right.
$$

such that $\widehat{\tau}_{k}^{p,(m)}$ is the smallest optimal stopping time after $t_{k}$ on the $m$ th path for the $p$-dimensional approximation. Hence, the conditional expectation $\mathbf{E}\left[O_{t_{k+1}} \mid \mathscr{F}_{t_{k}}\right]$ is computed by the following minimization problem:

$$
\theta_{k}^{p}=\arg \inf _{\theta \in \Theta} \mathbf{E}\left(\left|O_{\tau_{k+1}^{p}}-\phi_{k}^{p}\left(S_{t_{k}}, \theta\right)\right|^{2}\right),
$$

or in another way by its Monte Carlo approximation:

$$
\widehat{\theta}_{k}^{p, M}=\arg \inf _{\theta \in \Theta} \frac{1}{M} \sum_{m=1}^{M}\left|\phi_{k}^{p}\left(S_{t_{k}}^{(m)}, \theta\right)-O_{\widehat{\tau}_{k+1}^{p,(m)}}^{(m)}\right|^{2},
$$

such that $\Theta$ is a finite set in $\mathbb{R}$. The coefficients $\left(\widehat{\theta}_{k}^{p, M}\right)_{k=1, \cdots, N}$ give us convenient multiplicators of basis vectors in $L^{2}$ for determining the optimal policy of the system (2). Thus, the option price at time 0 is obtained as $U_{0}^{p}=\mathbf{E}\left(O_{\widehat{\tau}_{1}^{p}}\right)$ with $\tau_{1}$ $=\inf \left\{k \in\{1, \cdots, N\} \mid O_{k}=U_{k}\right\}$. Therefore, the Monte Carlo approximation is

$$
U_{0}^{p, M}=\sum_{m=1}^{M} O_{\widehat{\tau}_{1},(m)}^{(m)}
$$

2.3.1. The Inadequacy of the Model B\&S with the Real Financial Market. We break down the interval $[0, T]$ in $N$ periods of equal duration $\Delta t=T / N$ with $N$ large enough which makes it possible to discretize the interval in $t_{0}=0$, $\cdots t_{N}=N$.

$$
\log \frac{S\left(t_{j}\right)}{S\left(t_{j-1}\right)}=\left(r-\frac{1}{2} \sigma^{2}\right) \Delta t+\sigma W_{\Delta t} .
$$

This formula shows us that the log returns of the asset price follow a normal distribution, but as we can see in Figure 1, the empirical distribution of Microsoft's weekly $\log$ returns between 14/03/2016 and 17/03/2021 (https://fr .finance.yahoo.com/quote/MSFT/history? $\mathrm{p}=\mathrm{MSFT}$ ) has an excess kurtosis (i.e., density function of $\log \left(S\left(t_{j}\right) / S\left(t_{j-1}\right)\right)$ has a bigger pick around mean and heavy tails compared to the normal distribution, which means that $\log \left(S\left(t_{j}\right) / S(\right.$ $\left.t_{j-1}\right)$ ) values are more clustered around its mean and the probability that a jump occurs is more considerably presented) and negative skewness (i.e., the tails are shifted to the left compared to a symmetric normal distribution, which means that the decrease in the stock price outweighs the corresponding increase in Microsoft's log returns).

We can highlight other inconsistencies with the B\&S model like stochastic volatility with mean-reverting phenomena; indeed, in the real market volatility, it is not constant and we can also emphasize what is called "volatility clustering" (i.e., an uptick in volatility can be followed by higher volatility) (see [14]). In this paper, we content ourselves with constant volatility models.

\subsection{Forward Simulation for Pricing Asset Price}

Theorem 8 (first fundamental theorem of asset pricing). We price on a complete market if only if there exists a unique equivalent measure $\mathbb{Q}$.

In a complete market, the existence and uniqueness are satisfied. For further explanations, see [15]. In the Black 


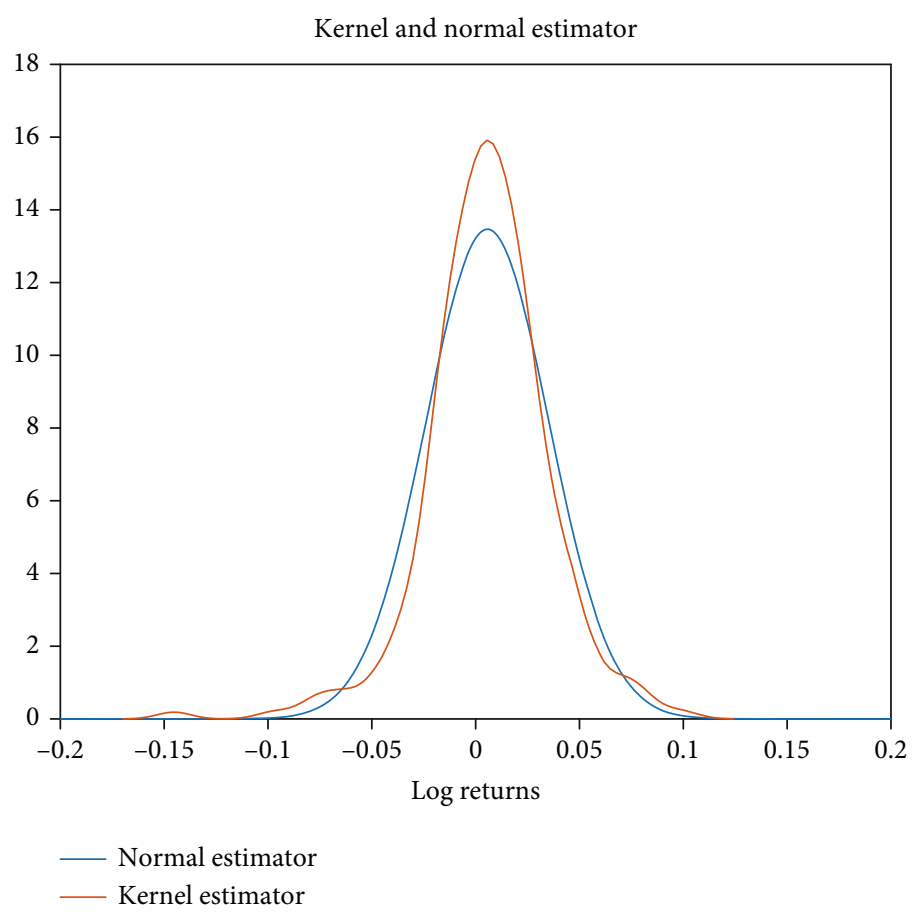

Figure 1: Kernel and normal estimator of Microsoft's log returns.

and Scholes model under the unique equivalent martingale (risk-neutral measure), the asset price follows the dynamics:

$$
d S_{t}=r S_{t} d t+\sigma S_{t} d B_{t}
$$

where $d B_{t}$ is the increment of the Brownian motion, $r$ the risk-free rate, and volatility $\sigma$.

Merton [6] notes that the source of the jumps can generally be caused by information specific to the firm or its industry, and in this case, they have a negligible impact on the rest of the market. The jump component then represents an unsystematic risk and will be uncorrelated with the market. The choice of the risk-neutral measure in the case of incomplete markets remains an open problem, although several lines of research have already been explored in this direction. In this regard, the reader can refer to the different works carried out by [11] or [16]. We will assume in the following that the dynamics of the underlying condition follows a diffusive model with jumps under a given risk-neutral measure $Q$. This measurement is arbitrary, and we will adjust the parameters; therefore, the evaluation obtained is as close as possible to the prices of market (see Section 3.3).

We suppose that the asset follows a Lévy jump-diffusion model:

$$
d S=r d t+\sigma d W_{t}+\mathrm{J}_{t} d N_{t},
$$

where $r$ is the risk-free rate, $\sigma$ is a constant volatility, and $J_{t}$ is the jump amplitude which flows an exponential law with parameter $\lambda_{1}=1$, and $d N_{t}$ is the increment of the compound Poisson process, $N_{T}$ is the number of jumps in $[0, T]$, and $T_{i}, i=1, \cdots, N_{T}$, is the time of jumps between 0 and $T$. We break down the interval $[0, T]$ in $N$ periods of equal duration $\Delta t=T / N$ to build the discretization $t_{0}=0, \cdots, t_{N}=T$, and therefore, we use the Euler schema to simulate $S(t)$ and $U($ $t, S(t))$ by discretizing equation (11) as

$$
S\left(t_{j+1}\right)-S\left(t_{j}\right)=r \Delta t+\sigma W_{\Delta t}+\sum_{i=0}^{N_{t_{j}}} J_{i}
$$

where $W_{t}$ is calculated from the cumulative of $W_{\Delta t} \sim N(0$, $\Delta t$ ); hence, a trajectory $\left.S_{t}\right|_{t=t_{1} \cdots t=t_{N}}$ is calculated from the application step-by-step of equation (12) as follows: first of all, we generate the Wiener process with a drift, and we use exponential laws for the duration between jumps $w_{i}=$ $T_{i+1}-T_{i}$ with its parameter $\lambda_{2}$ (on the average, we have 1/ $\lambda_{2}$ jumps in the meantime $[0, T]$ ), and as the case may be, we generate negative exponential law amplitudes for size jumps $J_{i}$ with its parameter $\lambda_{1}$ (i.e., losses which follow exponential laws) (see the example of Figure 2).

Theorem 9 (second fundamental theorem of asset pricing). In a free arbitrary market and under an appropriate equivalent measure $\mathbb{Q}$, the price at time $t$ is the conditional expected payout at $T$, i.e.,

$$
U_{t}=\mathbf{E}\left[e^{(T-t) r} U_{T} \mid \mathscr{F}_{t}\right]
$$

Hence, $e^{-r(T-t)} U_{t}$ is a martingale under $\mathbb{Q}$. Such an equivalent martingale measure is also called a risk-neutral measure because the return on the risk-free investment is the same as the expected return on the asset. 


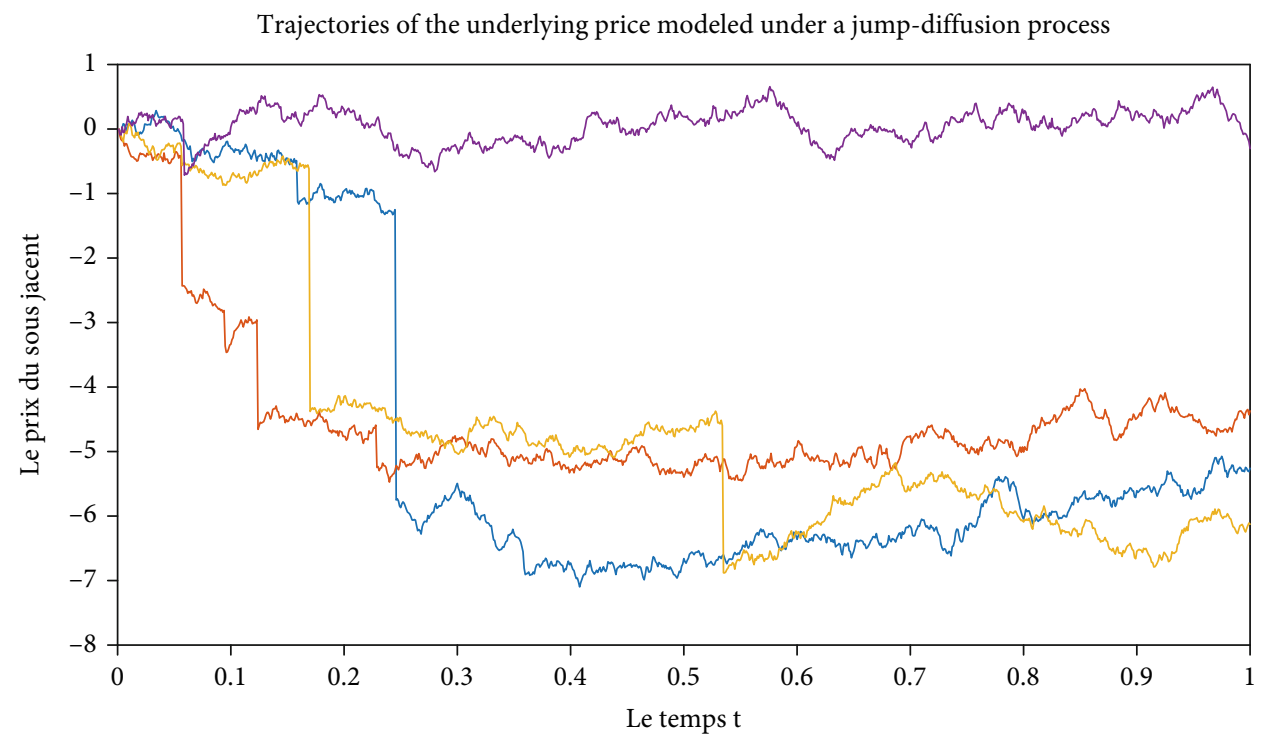

FIGURE 2: Modeling asset price paths under Lévy jump-diffusion $\lambda_{1}=1$ and $\lambda_{2}=1 / 4$.

\subsection{Least Square Regression (LS) Algorithm}

2.5.1. Methodology. We assume that $\pi=\left\{t_{0}=0, \cdots, t_{N}=T\right\}$ is the set of possible exercise times, $p$ is the number of regressors or the dimension of the $L^{2}$ basis, and $M$ is the number of paths. We aim to calculate the least square coefficients $\beta=\left(\alpha, \beta_{1}, \beta_{2}, \cdots, \beta_{p}\right)$. Let $\varphi=\left(\varphi_{1}, \varphi_{2}, \cdots, \varphi_{p}\right)$ be a basis of nonzero vectors in the Hilbert space $L^{2}$, and the orthogonal projection of $O\left(S_{t_{N}}\right)$ on $L^{2}\left(\sigma\left(S_{t_{N-1}}\right)\right)$ is the vector: $\alpha+\beta_{1} \varphi_{1}\left(S_{t_{N-1}}\right)+\beta_{2} \varphi_{2}\left(S_{t_{N-1}}\right)+\cdots+\beta_{p} \varphi_{p}\left(S_{t_{N-1}}\right)$. At time $t_{N-1}$ , we use an ordinary least square backward to regress $O\left(S_{N}\right)$ on the space generated by regressors $\varphi_{1}\left(S_{t_{N-1}}\right), \varphi_{2}\left(S_{t_{N-1}}\right), \cdots$ , $\varphi_{p}\left(S_{t_{N-1}}\right)$ by minimizing $\left(O\left(S_{t_{N}}\right)-\alpha-\beta_{1} S_{t_{N-1}}-\beta_{2} S_{t_{N-1}}\right)^{2}$; in this case, the continuation value becomes

$$
\begin{aligned}
\phi_{t_{N-1}}^{p}= & \mathbb{E}\left[e^{-r \Delta t} O\left(S_{t_{N}}^{i}\right) \mid S_{t_{N-1}}\right] \cong e^{-r \Delta t}\left(\alpha+\beta_{1} \varphi_{1}\left(S_{t_{N-1}}\right)\right. \\
& \left.+\beta_{2} \varphi_{2}\left(S_{t_{N-1}}\right)+\cdots+\beta_{p} \varphi_{p}\left(S_{t_{N-1}}\right)\right) .
\end{aligned}
$$

On each trajectory from $M^{\prime}$ in the money paths at time $t_{N-1}$, we obtain the least square coefficients $\widehat{\beta}_{t_{N-1}}$ by the least square estimator:

$$
\widehat{\beta}_{t_{N-1}}=\left(\begin{array}{c}
\alpha_{t_{N-1}} \\
\beta_{1, t_{N-1}} \\
\vdots \\
\beta_{p, t_{N-1}}
\end{array}\right)=\left(S^{T} S\right)^{-1} S^{T}\left(O\left(S_{T}^{1}\right) \cdots O\left(S_{T}^{M I}\right)\right),
$$

with

$$
S=\left(\begin{array}{ccc}
\varphi_{1}\left(S_{t_{N-1}}^{1}\right) & \cdots & \varphi_{1}\left(S_{t_{N-1}}^{M}\right) \\
\vdots & \ddots & \vdots \\
\varphi_{p}\left(S_{t_{N-1}}^{1}\right) & \cdots & \varphi_{p}\left(S_{t_{N-1}}^{M}\right)
\end{array}\right)
$$

Thus, we apply the equation (14) backward for $t_{t_{N-2}}$, $t_{N-3}, \cdots, t_{0}=0$.

\subsection{Convergence of Monte Carlo Simulation}

Theorem 10. Let $0 \leq j \leq N$, and we assume that $\varphi^{p}\left(S_{t_{j}}\right)=$ $\left(\varphi_{1}\left(S_{t_{j}}\right), \cdots, \varphi_{p}\left(S_{t_{j}}\right)\right)$ be a total basis in $L^{2}\left(\sigma\left(S_{t_{j}}\right)\right)$; then,

$$
\lim _{p \longrightarrow+\infty} \mathbf{E}\left(O_{\tau_{j}^{p}} \mid \mathscr{F}_{t_{j}}\right)=\mathbf{E}\left(O_{\tau_{j}} \mid \mathscr{F}_{t_{j}}\right) \text { in } L^{2}
$$

Proof. See [17].

This result can be proven theoretically but cannot be proven numerically, unlike the following convergence result. $\square \square$

Theorem 11. Assume that for $0 \leq j \leq N, P\left(\beta_{j} \varphi\left(S_{t_{j}}\right)=O_{t_{j}}\right)=0$ and the simulated $M$ paths are independent; then, $\lim$ $U_{0}^{p, M}=U_{0}^{p}$ almost surely.

Proof. First of all, we prove that for $0 \leq j \leq N,(1 / M) \sum_{k=1}^{M}$ $O_{\tau_{j}^{k, p, M}}^{k} \longrightarrow \mathbf{O}_{\tau_{j}^{p}}$ when $M \longrightarrow+\infty$ almost surely and then conclude our result (see [17]). The numerical illustration of this theorem is shown in Figures 3 and $4 . \square \square$

\section{Numerical Implementation}

3.1. Specific Example of the Least Square Regression Algorithm. We consider the case of $p=2$ to highlight the ordinary least square regression algorithm at time $t_{N-1}$; for example, we choose the following form of regressor functions: $\varphi_{1}\left(S_{t_{N-1}}\right)=S_{t_{N-1}}, \quad \varphi_{1}\left(S_{t_{N-1}}\right)=S_{t_{N-1}}^{2}, \quad$ and $\beta=\left(\alpha_{t_{N-1}}, \beta_{1, t_{N-1}}\right.$, $\left.\beta_{2, t_{N-1}}\right)$, an estimator of the three least square coefficients 


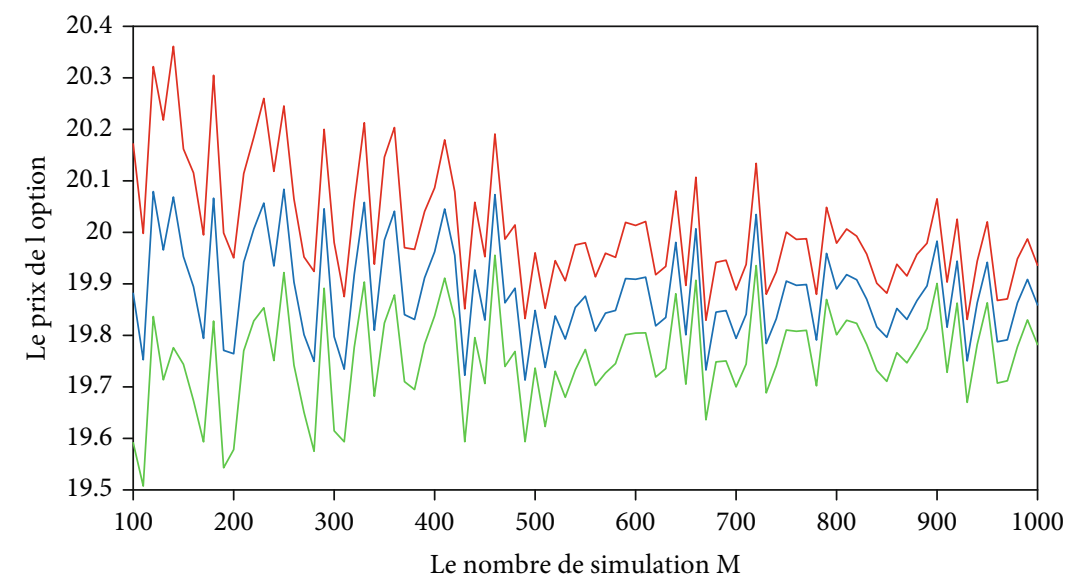

(a) $M$ from 100 to 1000 by 10 steps

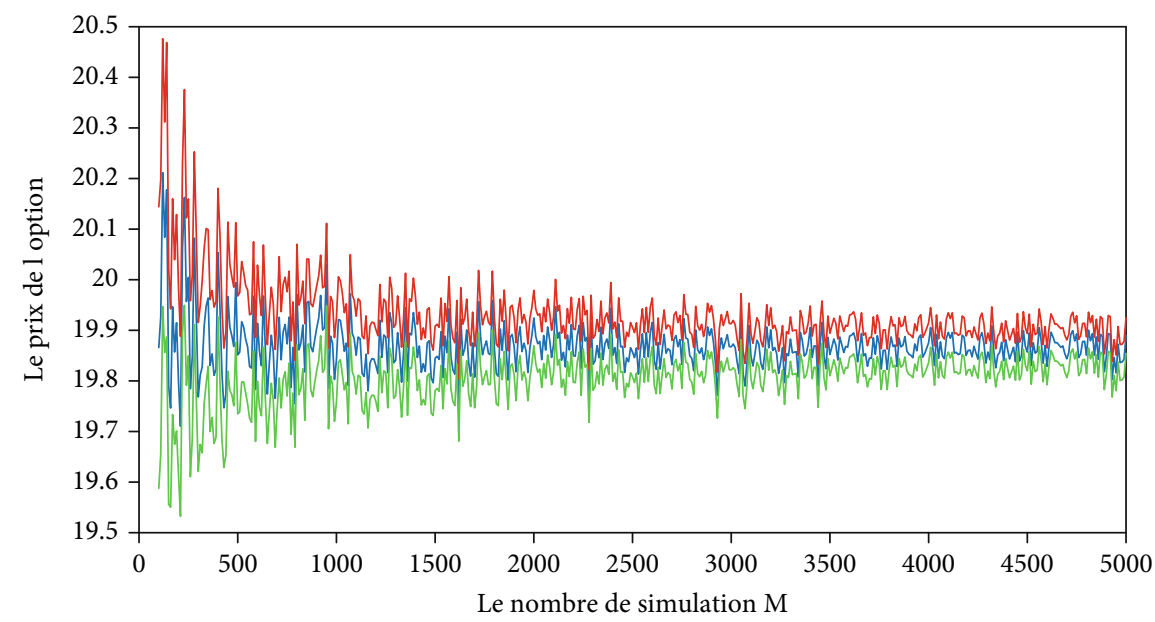

(b) $M$ from 100 to 5000 by 10 steps

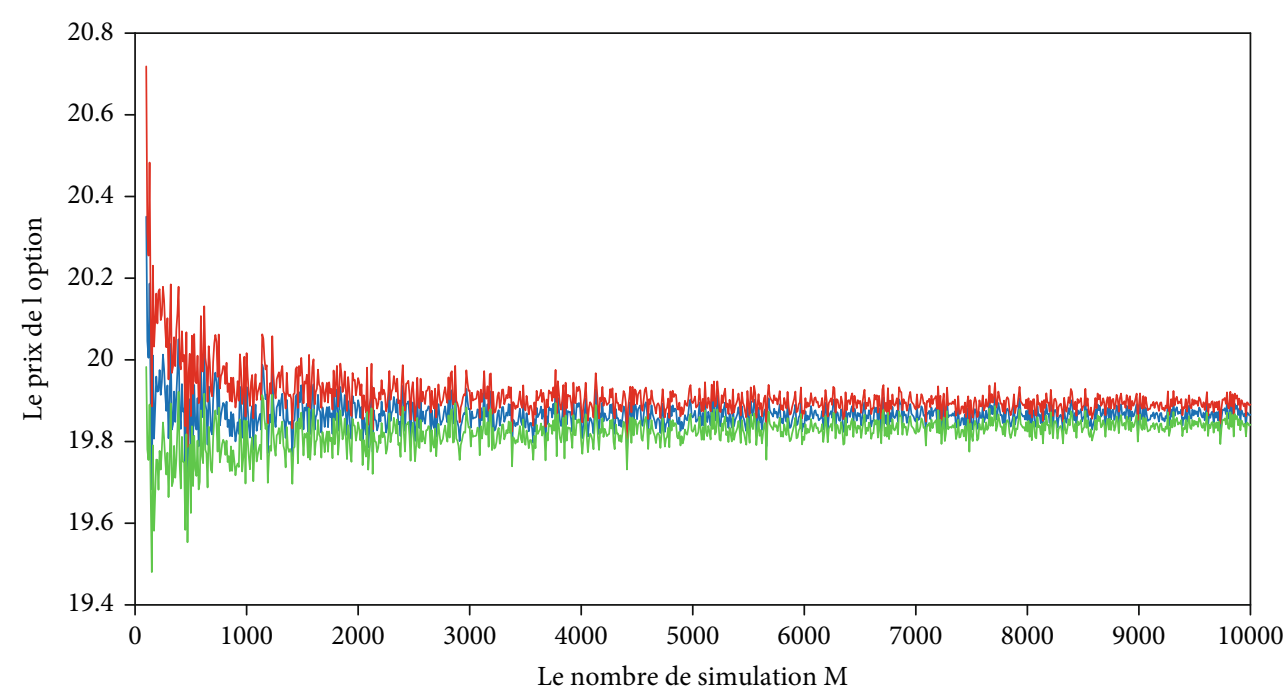

(c) $M$ from 100 to 10000 by 10 steps

FIGURE 3: Numerical convergence of Monte Carlo simulation in the least square algorithm under the B\&S asset price model. Confidence level in order of $95 \%$ (the estimated value of the American option in blue, upper bound in red, and lower bound in green). 
Convergence du modèle Longstaff Shwatrz, sous-jacent modélisé avec un processus de saut-diffusion

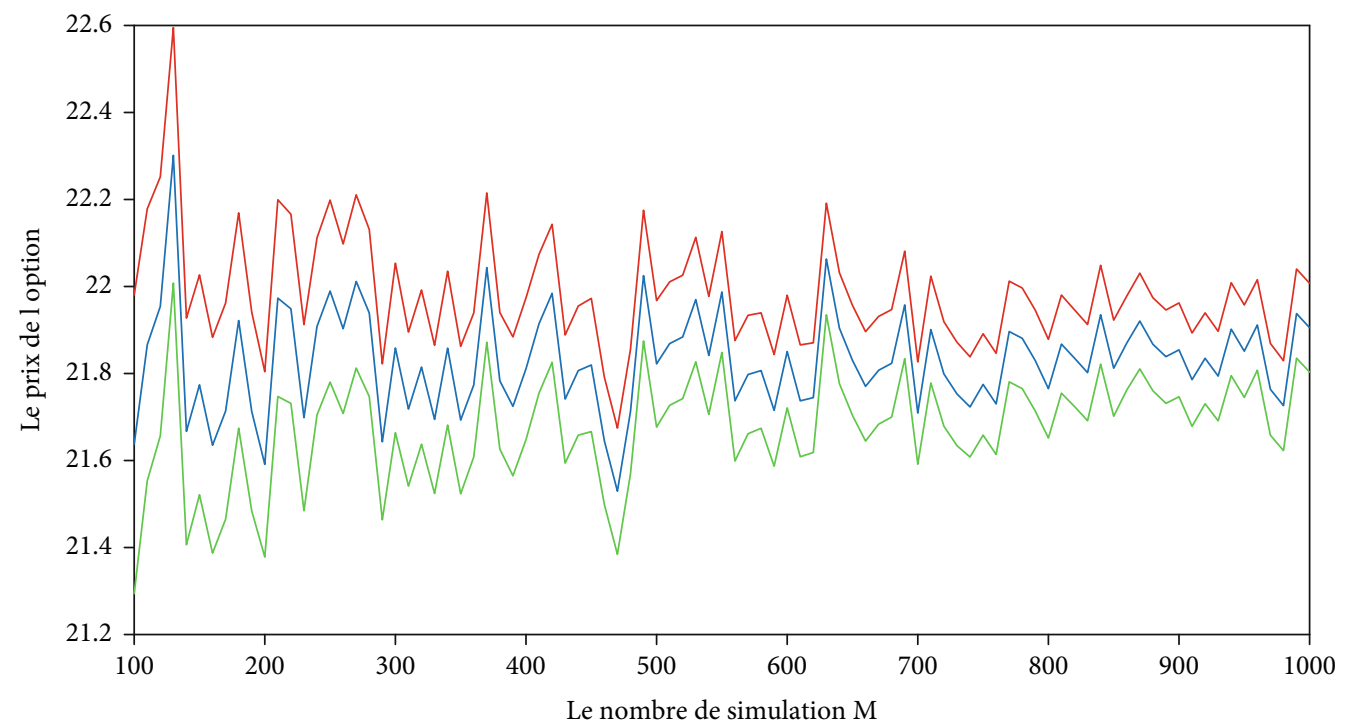

(a) $M$ from 100 to 1000 by 10 steps

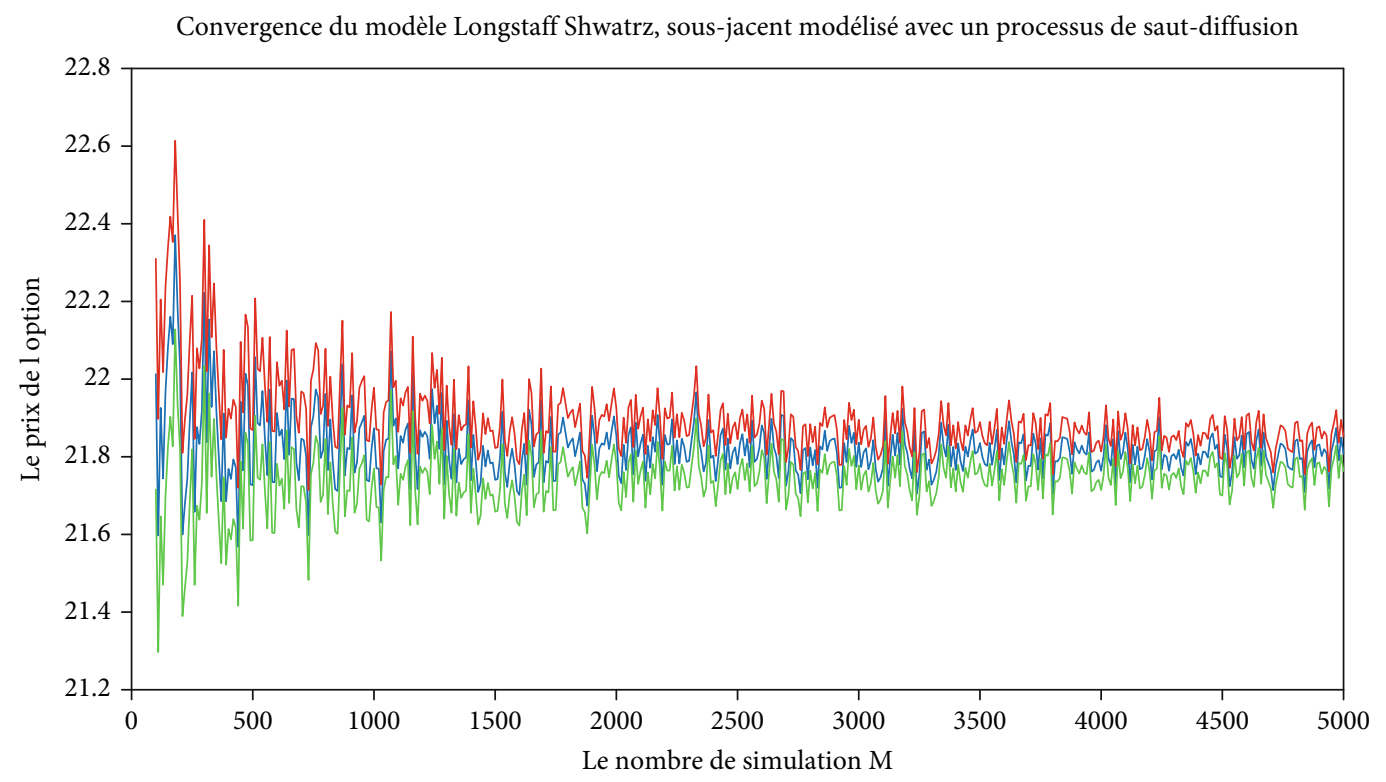

(b) $M$ from 100 to 5000 by 10 steps

Figure 4: Continued. 


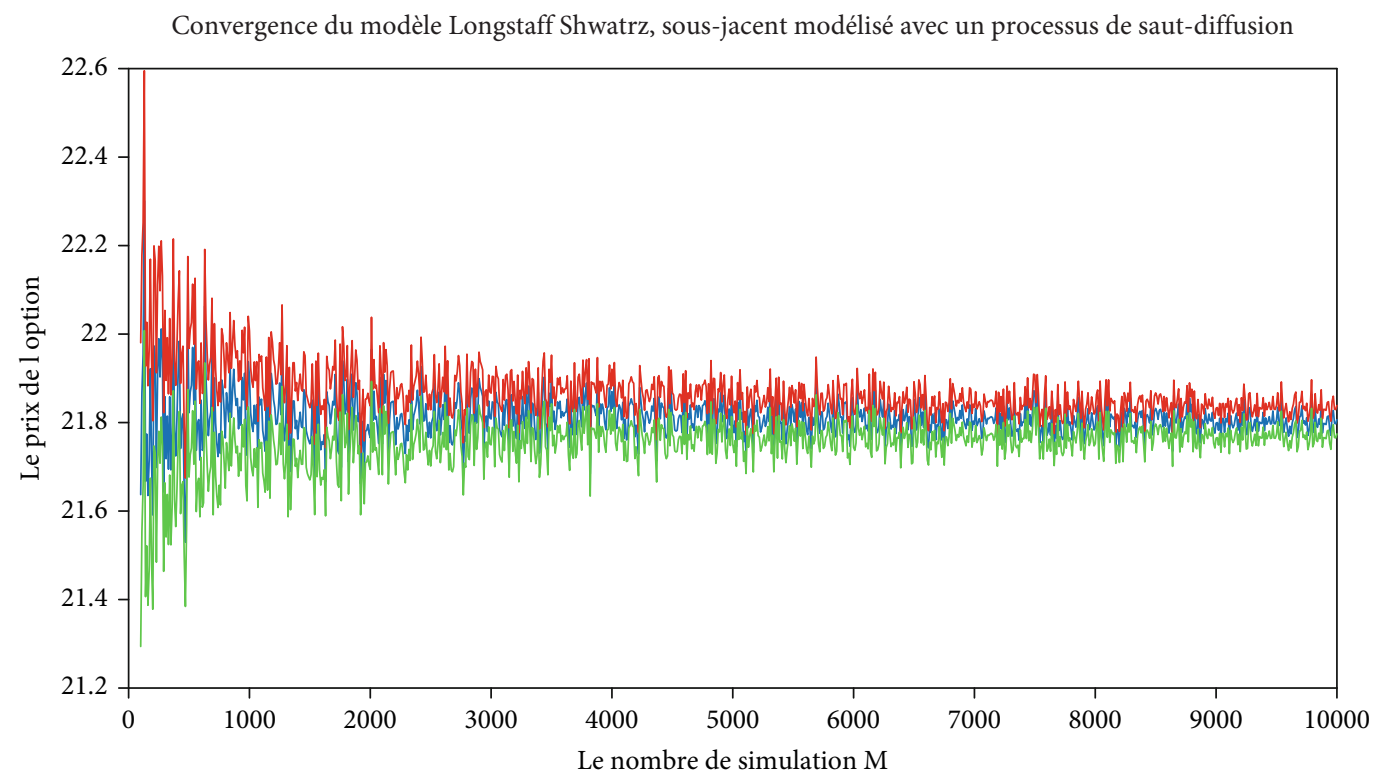

(c) $M$ from 100 to 10000 by 10 steps

Figure 4: Numerical convergence of Monte Carlo simulation in the least square algorithm under the Lévy jump-diffusion model. Confidence level in order of $95 \%$ (the estimated value of the American option in blue, upper bound in red, and lower bound in green).

which minimize the least square error:

$$
\sum_{i=1}^{M}{ }^{\prime}\left[O\left(S_{t_{N}}^{i}\right)-\alpha-\beta_{1} S_{t_{N-1}}^{i}-\beta_{2} S_{t_{N-1}}^{i 2}\right]^{2} .
$$

At each trajectory, the terminal value $O\left(S_{t_{N}}^{i}\right)$ equals to the terminal payoff of the option; as of date $t_{N-1}$, we estimate the continuation value if the trajectory is in the money and we reject if the trajectory is out of the money, so on each trajectory, we have $M^{\prime}$ couple $\left(S_{t_{N-1}}^{i}, O\left(S_{t_{N}}^{i}\right)\right)$ among $M$ which will explain $O\left(S_{t_{N-1}}^{i}\right)$ using the following regressors: $1, \varphi_{1}\left(S_{t_{N-1}}\right)=S_{t_{N-1}}, \varphi_{2}\left(S_{t_{N-1}}\right)=\left(S_{t_{N-1}}\right)^{2}$. According to equation (14) at $t_{N-1}$ on trajectory $i$, the continuation value is

$$
\begin{aligned}
\phi_{k}^{p}\left(t_{N-1}, S_{t_{N-1}}^{i}\right) & =\mathbb{E}\left[e^{-r \Delta t} O\left(S_{t_{N}}^{i}\right) \mid S_{t_{N-1}}^{i}=S_{t_{N-1}}\right] \\
& \cong e^{-r \Delta t}\left(\alpha_{t_{N-1}}-\beta_{1, t_{N-1}} S_{t_{N-1}}^{i}-\beta_{2, t_{N-1}}\left(S_{t_{N-1}}^{i}\right)^{2}\right) .
\end{aligned}
$$

We notice that the intrinsic value $e^{-r \Delta t} O\left(S_{t_{N}}^{i}\right)$ represents the discounted cash flows earned at time $t_{N}$ in the case where the option is not exercised at time $t_{N-1}$. Thus, we make a comparison between the payoff $O\left(S_{t_{N}}^{i}\right)$ and $\phi_{k}^{p}\left(t_{N-1}, S_{t_{N-1}}^{i}\right)$ on each trajectory if $O\left(S_{t_{N}}^{i}\right) \geq \phi_{k}^{p}\left(t_{N-1}, S_{t_{N-1}}^{i}\right)$ ; the early exercise of the option has therefore taken place at that time if it has not taken place previously. Hence, the value of option at date $t_{N-1}$ on trajectory $i$ is

$$
\left.U_{t_{N-1}}^{2, i}=\max \left(O\left(S_{t_{N-1}}^{i}\right)\right), \phi_{k}^{p}\left(S_{t_{N-1}}^{i}\right)\right)
$$

We do the same for all dates $t_{N-2}, \cdots, t_{0}$. Finally, an updated payoff is assigned to each trajectory $i$ : $\exp \left(-r \tau_{i}\right)$ $O_{\tau}^{i}$ or equals to 0 in the case of no exercise. Note that $\tau \in\left\{t_{0}\right.$ $\left.=0, \cdots, t_{N}=T\right\}$ is the first time when $O\left(S_{t_{N}}^{i}\right) \geq \phi_{k}^{p}\left(t_{N-1}\right.$, $\left.S_{t_{N-1}}^{i}\right)$, and as previously proven, this is an optimal exercising time; in the same logic as Section 2.3, we estimate the final price of the option at time $t_{0}$ by

$$
U_{0}^{2, M}=\sum_{i=1}^{M} \exp \left(-r \tau_{i}\right) O\left(S_{\tau_{i}}^{i}\right) .
$$

\subsection{Example of Pricing American Put and Validation on the} Real Market with the Microsoft Stock Option as an Example. We consider an American put with the following parameters: $\sigma=0.2$ the stock volatility, $S_{0}=80$ the price at time $t_{0}, T=1$ year of the maturity, $K=100$ the strike, and $r=$ $2 \%$ the interest rate. For the Lévy jump-diffusion model, we model the duration between jumps with exponential laws and its parameter which is $\lambda_{1}=1 / 4$, i.e., four jumps per one year. We also model the size of jumps with a negative exponential law with parameter $\lambda_{2}=1$; i.e., the size of asset price negatively jumps by 1 dollar.

According to the number of simulated paths, we illustrate the numerical convergence of the option price and we notice the asymptotic behavior when $M$ tends to have a large number. For the same algorithm "least square regression," we compare asymptotically the convergence between the B\&S model and the exponential Lévy jump-diffusion model (see Figure 5). We notice for this example that the B\&S model already stabilizes the size of the confidence interval when the $M$ tends to have $M=5000$, while the Lévy jump- 


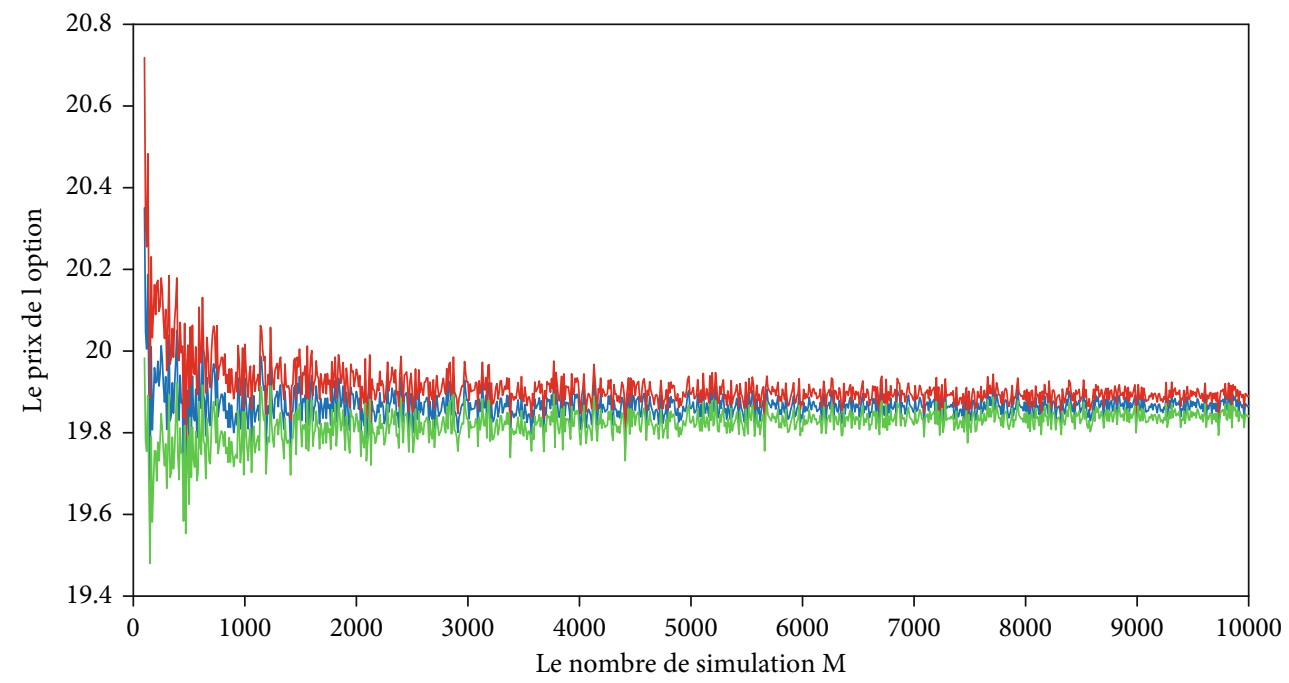

(a) Equilibrium price option under B\&S $U_{0}^{2, M}=19.8 \$$

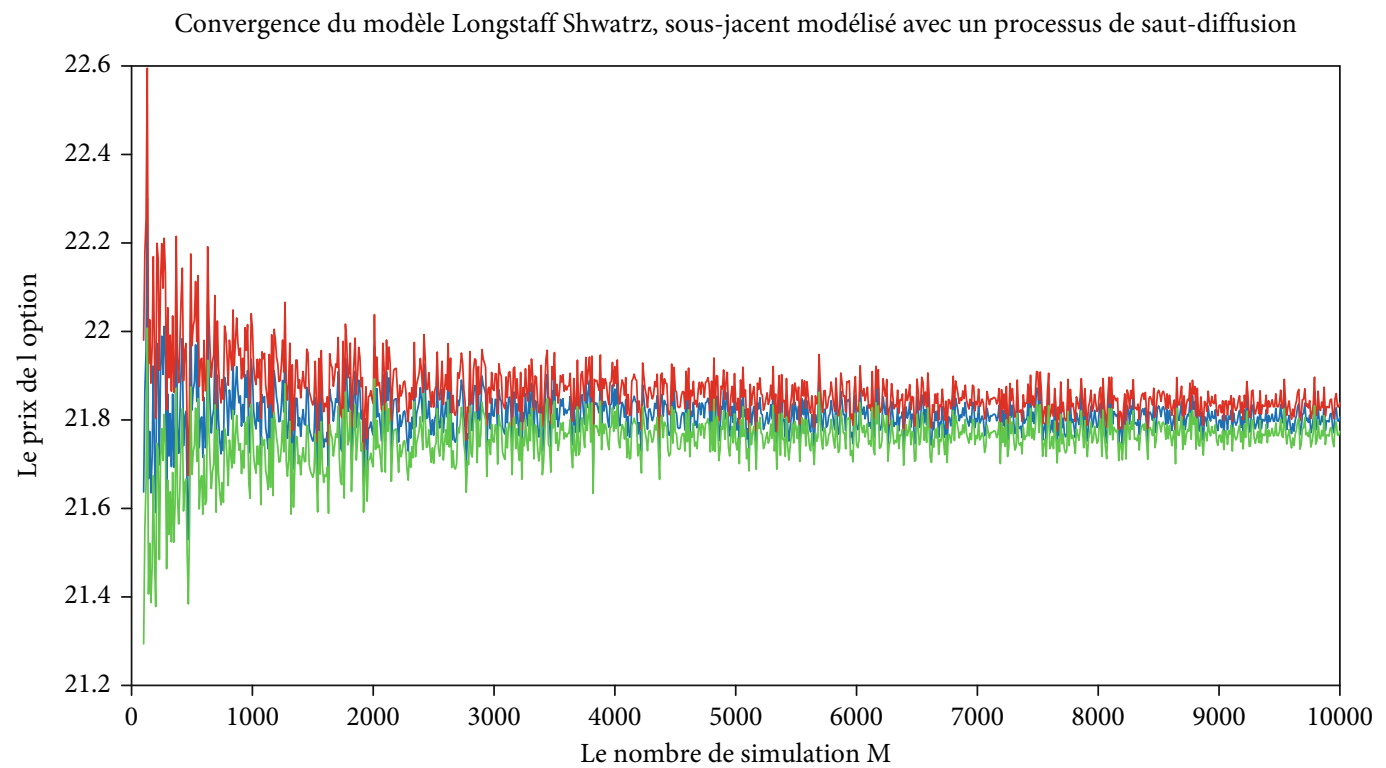

(b) Equilibrium price option under the Lévy jump-diffusion process $U_{0}^{2, M}=21.82 \$$

Figure 5: Asymptotic convergence of the least square algorithm $M$ from 100 to 10000 . Confidence level in order of $95 \%$ (the estimated value of the American option in blue, upper bound in red, and lower bound in green).

TABLE 1: Confidence interval according to the number of sampling.

\begin{tabular}{lcccccc}
\hline Trajectories & Mean BS & Min BS & Max BS & Mean jump-dif & Min jump-dif & Max jump-dif \\
\hline$M=1000$ & 19.8883 & 19.8103 & 19.9663 & 21.7427 & 21.6420 & 21.8433 \\
$M=5000$ & 19.8414 & 19.8061 & 19.8768 & 21.8000 & 21.8000 & 21.77746 \\
$M=8000$ & 19.8514 & 19.8011 & 19.8721 & 21.8270 & 21.7950 & 21.8401 \\
$M=10000$ & 19.8528 & 19.8079 & 19.8711 & & 21.8390 \\
\hline
\end{tabular}

TABLE 2: Time consumption (MATLAB R2019a program).

\begin{tabular}{lcc}
\hline Number of sampling & B\&S & Jump-diffusion \\
\hline 100 to 1000 by 10 steps & $24.542 \mathrm{~s}$ & $40.544 \mathrm{~s}$ \\
100 to 5000 by 10 steps & $597.303 \mathrm{~s}$ & $1163.004 \mathrm{~s}$ \\
100 to 10000 by 10 steps & $16305.825 \mathrm{~s}$ & $57092.476 \mathrm{~s}$ \\
\hline
\end{tabular}

diffusion started to stabilize the confidence interval around $M=8000$; we come back to this phenomenon of the robustness of B\&S in terms of stabilization of the size of the confidence interval in an early number of simulated paths compared to the Lévy jump-diffusion model (see Table 1). Table 2 shows the speed of convergence in terms of time. 
TABLE 3: MSFT put option traded on $19 / 03 / 2021$ at a price of $S_{0}=231.02$, expired on 18/03/2021 $(r=4 \%)$ (from https://fr.finance.yahoo .com/quote/MSFT?p=MSFT).

\begin{tabular}{|c|c|c|c|c|c|c|}
\hline Strike & Option price & Implied volatility & B\&S & Error & Jump-diffusion & Error \\
\hline 80 & 0.63 & $25 \%$ & 0 & 0.63 & 0.0338 & 0.592 \\
\hline 100 & 1.1 & $12.5 \%$ & 0 & 1.1 & 0.1557 & 0.9443 \\
\hline 145 & 3.58 & $12.5 \%$ & 0 & 3.58 & 1.2832 & 2.2968 \\
\hline 150 & 4.05 & $6.25 \%$ & 0 & 4.05 & 1.8354 & 2.2141 \\
\hline 160 & 5.4 & $6.25 \%$ & 0 & 5.4 & 3.0275 & 2.3725 \\
\hline 185 & 9.81 & $6.25 \%$ & 0 & 9.81 & 8.5283 & 1.2817 \\
\hline 210 & 17.8 & $1.56 \%$ & 0 & 17.8 & 21.7962 & 3.9962 \\
\hline 215 & 19.8 & $1.56 \%$ & 0 & 19.8 & 24.853 & 5.053 \\
\hline 220 & 21.95 & $0.78 \%$ & 0 & 21.95 & 21.5588 & 0.3912 \\
\hline 225 & 24.25 & $0.78 \%$ & 0 & 24.25 & 25.5089 & 1.2589 \\
\hline 230 & 26.6 & $0.05 \%$ & 0 & 26.6 & 27.7062 & 1.1062 \\
\hline 255 & 41.48 & $0 \%$ & 23.6801 & 17.7999 & 42.5198 & 5.4856 \\
\hline 270 & 51.45 & $0 \%$ & 38.6561 & 12.7939 & 56.9356 & 5.4856 \\
\hline 275 & 55.2 & $0 \%$ & 43.6481 & 11.5519 & 57.935 & 4.485 \\
\hline 305 & 80.53 & $0 \%$ & 73.6002 & 6.9298 & 71.3296 & 9.2004 \\
\hline \multirow[t]{2}{*}{355} & 126.68 & $0 \%$ & 123.5202 & 3.1598 & 121.6777 & 5.0023 \\
\hline & & & Mean error & 11.7003 & Mean error & 2.8106 \\
\hline
\end{tabular}

According to the central limit theorem and because of the independence of trajectories, the sum of the option price of each path in the Monte Carlo approximation can be estimated by a normal distribution $\mathscr{N}(0, \sigma / \sqrt{M})$ (Monte Carlo estimator unbiased); hence, $\left(U_{0}^{M} /(\sigma / \sqrt{M})\right) \sim \mathcal{N}(0,1)$.

We propose an estimator of the theoretical tolerance range with a confidence level of $95 \%$ by subtracting the upper and lower bounds of the confidence interval according to $M$.

$$
\widehat{I}_{\text {model }}=\frac{\text { upper }_{\text {model }}^{M}-\text { lower }_{\text {model }}^{M}}{2}=2 * 1.96 * \frac{\sigma}{\sqrt{M}} .
$$

For example, $M=10000 \widehat{I}_{\text {levy }}=(21.8390-21.7950) / 2=$ 0.022 which is a very small range error, so theoretically the price option belongs to $[21.82-0.022,21.849+0.022]$ with a confidence level of $95 \%$.

Now, the question that arises is whether B\&S or exponential Lévy is more effective in predicting prices in the real market.

3.3. Validation of the B\&S Model Compared to the Exponential Lévy Model on a Real Market with the Microsoft Option as an Example. We notice in Table 3 that BS defaults below the spot 230 at time $0, B \& S$ suppose that all paths are out of the money in the meantime $[0, T]$ throughout all strikes below 230 [[80,230]], and this comes from the fact that in a complete market, continuous asset prices $S_{t}$ with drift are unlikely to fall below 230 from $S_{0}=$ 231.02; hence, the American put price is equal to 0. On the other hand, jump-diffusion with negative jumps predicts more efficiently than B\&S from $S_{0}=80$ up to $S_{0}=230$, and the prediction error remains around 1 dollar. In our case, calibration of the parameters in this spot range is as follows: $\lambda_{1}=-8$ which corresponds to the expectation of losses "following the exponential law" of the Microsoft share chosen as the average of share losses between 2020 and 2021" and $\lambda_{2}$ which represents the expectation of losses with jump occurrence, chosen equal to $1 / 3$. From strike $k=255$ to 275 with some calibration (i.e., the assumption of a small positive parameter of jump size "profit jumps" $\lambda_{1}=1$ ) Lévy's jumpdiffusion model outperforms B\&S. For $k=305,355, \mathrm{~B} \& \mathrm{~S}$ is more precise, and it seems that the hypothesis of continuous asset price's market is more effective for these values. Generally, the mean error of the jump-diffusion model is in the order of 2.8 dollars; on the other hand, $\mathrm{B} \& \mathrm{~S}$ mean error is equal to 11.7003, which shows that the Lévy jumpdiffusion model outperforms widely B\&S to validate our example on the real market.

Remark 12. The use of the improved exponential Lévy jumpdiffusion model with hybrid jumps "loss and profit jumps like in Kou model jumps which follows a generalized Laplace nonsymmetric law" [18] can slightly improve our results.

\section{Conclusion}

In this paper, we have presented a comparison between the least square algorithm under B\&S and exponential Lévy jump-diffusion processes in terms of time-consuming and asymptotic convergence properties depending on the number of simulated trajectories, and we pointed out that B\&S converges faster and its option price variance is less volatile than that of Lévy's jump-diffusion model which makes the calculation more easier and faster, which is useful in the case of multidimensional underlying assets. However, as we have pointed out in Sections 2.3.1 and 3.3, the Lévy model stays 
more adequate than B\&S in our validation example on Microsoft's put option. We have also proposed a simple method to estimate theoretical tolerated range according to the confidence level in Section 3.2. We also noted that by varying the number of paths, we numerically highlighted the equilibrium option price.

More calibrations can perform better our exponential Lévy jump-diffusion model as done in the context European option of the S\&P100 index in this paper [16].

Recent work on machine learning and the parallelism method for solving BSDEs opens a horizon as in [19] where the author tried to cluster the computational method using machine learning instead of using a brute force approximation of the true solution, which is time-consuming as in $[20,21]$.

\section{Appendix}

\section{A. Properties of the snell envelope}

(1) At time $t=T$, it is obvious that $U_{T}=O_{T}$

(2) We consider $t \in\left\{t_{0}=0, \cdots, t_{n-1}\right\}$; it is obvious that $U_{t_{j}} \geq O_{t_{j}}$ because of the definition

$U_{t_{j}} \geq \mathbf{E}\left[O_{t_{j}} \mid \mathscr{F}_{t_{j}}\right]$ because $t_{k}$ is the $F_{t_{k}}$ stopping time; consequently, $U_{t_{j}} \geq O_{t_{j}}$.

On the other hand,

$$
\begin{aligned}
\mathbf{E}\left[U_{t_{j+1}} \mid \mathscr{F}_{t_{j}}\right] & =\mathbf{E}\left[\sup _{\tau \in \Gamma_{t_{j+1}}} \mathbf{E}\left[O_{\tau} \mid \mathscr{F}_{t_{j+1}}\right] \mid \mathscr{F}_{t_{j}}\right] \\
& =\sup _{\tau \in \Gamma_{t_{j+1}}} \mathbf{E}\left[O_{\tau} \mid \mathscr{F}_{t_{j}}\right] \\
& \leq \sup _{\tau \in \Gamma_{t_{j}}} \mathbf{E}\left[O_{\tau} \mid \mathscr{F}_{t_{j}}\right]=U_{t_{k}} .
\end{aligned}
$$

Hence, $U_{t_{j}} \geq \max \left(O_{t_{j}}, \mathbf{E}\left[U_{t_{j+1}} \mid \mathscr{F}_{t_{j}}\right]\right)$.

Conversely, $\mathbf{E}\left[O_{\tau} \mid \mathscr{F}_{t_{j}}\right]=O_{t_{j}} \mathbf{1}_{\tau=t_{j}}+\mathbf{E}\left[O_{\tau} \mid \mathscr{F}_{t_{j}}\right] \mathbf{1}_{\tau \geq t_{j+1}}$.

By adding $\sup _{\tau \in \Gamma_{t_{j}}}$ in both sides of the equation, we have

$$
\begin{aligned}
U_{t_{j}} & =O_{t_{j}} \mathbf{1}_{\tau=t_{j}}+\mathbf{E}\left[\sup _{\tau \in \Gamma_{t_{j+1}}} \mathbf{E}\left[O_{\tau} \mid \mathscr{F}_{t_{j+1}}\right] \mid \mathscr{F}_{t_{j}}\right] \mathbf{1}_{\tau \geq t_{j+1}} \\
& =O_{t_{j}} \mathbf{1}_{\tau=t_{j}}+\mathbf{E}\left[U_{t_{j+1}} \mid \mathscr{F}_{t_{j}}\right] \mathbf{1}_{\tau \geq t_{j+1}} .
\end{aligned}
$$

Then, $U_{t_{j}}=O_{t_{j}} \mathbf{1}_{\tau=t_{j}}+\mathbf{E}\left[U_{t_{j+1}} \mid \mathscr{F}_{t_{j}}\right] \mathbf{1}_{\tau \geq t_{j+1}} \leq \max \left(O_{t_{j}}, \mathbf{E}[\right.$ $\left.\left.U_{t_{j+1}} \mid \mathscr{F}_{t_{j}}\right]\right)$.

(3) It is obvious that $U$ is a supermartingale. Assume that $W$ is another supermartingale which dominates $\left(O_{t_{j}}\right)$; consequently,

$$
\begin{aligned}
W_{t_{j}} \geq \mathbf{E}\left[W_{t_{j+1}} \mid \mathscr{F}_{t_{j}}\right] \geq \mathbf{E}\left[\sup _{\tau \in \Gamma_{t_{j+1}}} \mathbf{E}\left[O_{\tau} \mid \mathscr{F}_{t_{j+1}}\right] \mid \mathscr{F}_{t_{j}}\right] \\
=\mathbf{E}\left[U_{t_{j+1}} \mid \mathscr{F}_{t_{j}}\right] \\
\quad \text { and } W_{t_{j}} \geq O_{t_{j}} ; \text { hence, } W_{t_{j}} \geq \max \left(O_{t_{j}}, \mathbf{E}\left[U_{t_{j+1}} \mid \mathscr{F}_{t_{j}}\right]\right) \\
=U_{t_{j}} .
\end{aligned}
$$

\section{B. Characterization of the optimal stopping time}

(i) Let $\tau$ be a stopping time such that $U_{\tau \wedge t_{j}}$ is a martingale $t_{j} \in\left\{t_{0}=0, \cdots, t_{n}=T\right\}$ and $U_{\tau}=O_{\tau}$; we aim to prove that $\tau$ is optimal. $U_{t_{0}}=U_{t_{0} \wedge \tau}=\mathbf{E}\left(U_{T \wedge \tau} \mid \mathscr{F}_{0}\right)$ $=\mathbf{E}\left(U_{\tau} \mid \mathscr{F}_{0}\right)=E\left(O_{\tau} \mid \mathscr{F}_{0}\right)$.

Let $\tau \in \Gamma_{0}$ (i.e., the set of all stopping time in $\left\{t_{0}=0, \cdots\right.$, $\left.\left.t_{n}=T\right\}\right)$ be an ordinary stopping time, since $U_{\tau}$ is a supermartingale which dominates $O$, so $U_{t_{0}} \geq \mathbf{E}\left(U_{T \wedge \tau} \mid \mathscr{F}_{t_{0}}\right)=\mathbf{E}\left(U_{\tau} \mid\right.$ $\left.\mathscr{F}_{t_{0}}\right)=\mathbf{E}\left(O_{\tau} \mid \mathscr{F}_{t_{0}}\right)$.

Hence, $U_{t_{0}}=\sup _{\tau \in \Gamma_{t_{0}}} \mathbf{E}\left(O_{\tau} \mid \mathscr{F}_{t_{0}}\right)$.

Conversely, assume that $\tau$ is an optimal stopping time:

$$
U_{t_{0}}=\sup _{\tau^{\prime} \in \Gamma_{t_{0}}} \mathbf{E}\left(O_{\tau^{\prime}} \mid \mathscr{F}_{t_{0}}\right)=\mathbf{E}\left(O_{\tau} \mid \mathscr{F}_{t_{0}}\right) \leq \mathbf{E}\left(U_{\tau} \mid \mathscr{F}_{t_{0}}\right) \leq U_{t_{0}},
$$

because $U$ is a supermartingale and $U$ dominates $O$. So $\mathbf{E}\left(O_{\tau} \mid \mathscr{F}_{t_{0}}\right)=\mathbf{E}\left(U_{\tau} \mid \mathscr{F}_{t_{0}}\right) \Rightarrow \mathbf{E}\left[O_{\tau}\right]=\mathbf{E}\left[U_{\tau}\right] \Rightarrow O_{\tau}=U_{\tau}$

because the two random variables are continuous and positive.

Furthermore, if $t_{j}>\tau$, then $U_{t_{0}} \geq \mathbf{E}\left(U_{t_{j} \wedge \tau} \mid \mathscr{F}_{t_{0}}\right) \geq \mathbf{E}\left(U_{\tau}\right.$ $\left.\mid \mathscr{F}_{t_{0}}\right)=U_{t_{0}}$ because $U_{t_{j} \wedge \tau} \geq \mathbf{E}\left[U_{\tau} \mid \mathscr{F}_{t_{j}}\right]$, since $\mathbf{E}\left(U_{\tau} \mid \mathscr{F}_{t_{0}}\right)$ $=\mathbf{E}\left(\mathbf{E}\left(U_{\tau} \mid \mathscr{F}_{t_{j}}\right) \mid \mathscr{F}_{t_{0}}\right)$.

Therefore, $\quad \mathbf{E}\left(U_{t_{j} \wedge \tau} \mid \mathscr{F}_{0}\right)=\mathbf{E}\left(\mathbf{E}\left(U_{\tau} \mid \mathscr{F}_{t_{0}}\right) \mid \mathscr{F}_{j}\right) \Rightarrow U_{t_{j} \wedge \tau}$ $=\mathbf{E}\left(U_{\tau} \mid \mathscr{F}_{t_{j}}\right)$. Thus, $U_{t_{j} \wedge \tau}$ is a martingale.

Otherwise, if $t_{j}<\tau, U_{t_{j} \wedge \tau}=U_{\tau}$, since $\mathbf{E}\left[U_{\tau} \mid \mathscr{F}_{t_{0}}\right]=\mathbf{E}[\mathbf{E}[$ $\left.\left.U_{t_{j}} \mid \mathscr{F}_{\tau}\right] \mid \mathscr{F}_{t_{0}}\right]$, so $U_{\tau}=\mathbf{E}\left[U_{t_{j}} \mid \mathscr{F}_{\tau}\right]$.

\section{The optimal stopping time of the Longstaff and Schwartz algorithm}

(i) Is $U_{\tau \wedge t_{j+1}}$ a martingale?

$$
\begin{aligned}
\mathbf{E}\left[U_{\tau \wedge t_{j+1}} \mid \mathscr{F}_{t_{j}}\right] & =\mathbf{E}\left[U_{t_{j+1}} \mid \mathscr{F}_{t_{j}}\right] \mathbf{1}_{\tau \geq t_{j+1}}+\mathbf{E}\left[U_{\tau} \mid \mathscr{F}_{t_{j}}\right] \mathbf{1}_{\tau<t_{j+1}} \\
& =U_{t_{j}} \mathbf{1}_{\tau>t_{j}}+U_{\tau} \mathbf{1}_{\tau \leq t_{j}}=U_{\tau \wedge t_{j}},
\end{aligned}
$$

because $\tau=\min \left\{k \geq 0 \mid U_{k}=O_{k}\right\}$, and for $t_{j}$ such that $\tau>t_{j}$, we have $U_{t_{j}}=\mathbf{E}\left[U_{t_{j+1}} \mid \mathscr{F}_{t_{j}}\right]$, and in $\left\{t_{j} \mid \tau\right.$ 
$\left.\leq t_{j}\right\}, \quad U_{\tau}$ is $\mathscr{F}_{t_{j}}$ measurable. Thus, $U_{\tau \wedge t_{j}}$ is a martingale.

(ii) Is $\tau=\min \left\{k \geq 0 \mid U_{k}=O_{k}\right\}$ optimal?

As shown previously, $U_{t \wedge \tau}$ is a martingale, so $U_{0}=$ $U_{0 \wedge \tau}=\mathbf{E}\left(U_{T \wedge \tau} \mid \mathscr{F}_{0}\right)=\mathbf{E}\left(U_{\tau} \mid \mathscr{F}_{0}\right)=E\left(O_{\tau} \mid \mathscr{F}_{0}\right)$, since $U_{0}=$ $\sup _{\tau}{ }^{\prime} \in \Gamma_{0} \mathbf{E}\left(O_{\tau}{ }^{\prime} \mid \mathscr{F}_{0}\right)$. Hence, $E\left(O_{\tau} \mid \mathscr{F}_{0}\right)=\sup _{\tau}{ }^{\prime} \in \Gamma_{0} \mathbf{E}\left(O_{\tau}{ }^{\prime} \mid\right.$ $\left.\mathscr{F}_{0}\right)$, and therefore, $\tau$ is optimal.

\section{Data Availability}

The MSFT share price and price option data used to support the findings of this study are available from the corresponding author upon request.

\section{Conflicts of Interest}

The authors declare that there is no conflict of interest.

\section{References}

[1] M. Scholes, "The pricing of options and corporate," The Journal of Political Economy, vol. 81, pp. 637-654, 1973.

[2] P. Jorion, "On jump processes in the foreign exchange and stock markets," The Review of Financial Studies, vol. 1, no. 4, pp. 427-445, 1988.

[3] E. Jondeau, S.-H. Poon, and M. Rockinger, Financial Modeling under Non-Gaussian Distributions, Springer Science \& Business Media, 2007.

[4] E. F. Fama, "Portfolio analysis in a stable Paretian market," Management Science, vol. 11, no. 3, pp. 404-419, 1965.

[5] B. Mandelbrot, "New methods in statistical economics," Journal of Political Economy, vol. 71, no. 5, pp. 421-440, 1963.

[6] R. C. Merton, "Option pricing when underlying stock returns are discontinuous," Journal of financial economics, vol. 3, no. 1-2, pp. 125-144, 1976.

[7] R. Breen, "The accelerated binomial option pricing model," Journal of Financial and Quantitative Analysis, vol. 26, no. 2, p. 153, 1991.

[8] J. Hull and A. White, "Valuing derivative securities using the explicit finite difference method," Journal of Financial and Quantitative Analysis, vol. 25, no. 1, p. 87, 1990.

[9] J. F. Carriere, "Valuation of the early-exercise price for options using simulations and nonparametric regression," Insurance: Mathematics and Economics, vol. 19, no. 1, pp. 19-30, 1996.

[10] F. A. Longstaff and E. S. Schwartz, "Valuing American options by simulation: a simple least-squares approach," The Review of Financial Studies, vol. 14, no. 1, pp. 113-147, 2001.

[11] P. Tankov, E. Voltchkova, and R. Cont, "Option pricing models with jumps: integro-differential equations and inverse problems," European Congress on Computational Methods in Applied Sciences and Engineering, 2004.

[12] T. Fujiwara and Y. Miyahara, "The minimal entropy martingale measures for geometric Lévy processes," Finance and Stochastics, vol. 7, no. 4, pp. 509-531, 2003.

[13] J. N. Tsitsiklis and B. van Roy, "Regression methods for pricing complex American-style options," IEEE Transactions on Neural Networks, vol. 12, no. 4, pp. 694-703, 2001.

[14] M. Klimek et al., "Pricing American options using Lévy processes and Monte Carlo simulations," 2015.
[15] T. Björk, Arbitrage Theory in Continuous Time, Oxford university press, 2009.

[16] F. M. Quittard-Pinon and R. Randrianarivony, "Calibrage d'options pour trois modèles mixtes diffusions et sauts," Finance, vol. 29, no. 2, pp. 103-130, 2008.

[17] E. Clément, D. Lamberton, and P. Protter, "An analysis of a least squares regression method for American option pricing," Finance and Stochastics, vol. 6, no. 4, pp. 449-471, 2002.

[18] S. G. Kou, “A jump-diffusion model for option pricing," Management Science, vol. 48, no. 8, pp. 1086-1101, 2002.

[19] L. Zhang, "A clustering method to solve backward stochastic differential equations with jumps," Journal of Mathematical Finance, vol. 10, no. 1, pp. 1-9, 2020.

[20] C. Beck, W. E, and A. Jentzen, "Machine learning approximation algorithms for high-dimensional fully nonlinear partial differential equations and second-order backward stochastic differential equations," Journal of Nonlinear Science, vol. 29, no. 4, pp. 1563-1619, 2019.

[21] W. E, J. Han, and A. Jentzen, "Deep learning-based numerical methods for high-dimensional parabolic partial differential equations and backward stochastic differential equations," Communications in Mathematics and Statistics, vol. 5, no. 4, pp. 349-380, 2017. 